\title{
Morphological Operation based Vehicle Number Plate Detection
}

\author{
N. Eswar \\ ${ }^{1} \mathrm{M}$. Tech, \\ Department of Electronics and Communication \\ Engineering, \\ SV University, Tirupathi
}

\begin{abstract}
Segmenting the Region Of Interest (ROI) from an image can be used for Vehicle number Plate detection. In this work a method based on convolution neural network is proposed for Vehicle number Plate detection.The accuracy of the method is impreoved and compared with the existing menthod.
\end{abstract}

Key Words : CNN, ROI

\section{INTRODUCTION:}

Nowadays the density of traffic is increasing rapidly. In order to monitor the traffic, thetraditional methods sometimes fail to provide an optimal solution. In order toovercome this problem, an automatic vehicle number plate detection technique shouldbe developed. Automatic VLP detection systems provide more effective and technicaladvantages than the traditional traffic monitoring systems. Moreover, additionaltraffic information can be obtained from images including vehicle classification; linechanges and a single camera can monitor multiple lines and can simultaneously readinformation about the vehicle.A number of methods regarding VLP detectionhave been introduced so far, but optimization and increasing their accuracy rate is a must. The reason behind, the accuracy rate of some license plate detection algorithms are not up to the mark and some other algorithms fail to detect the ROI in a dynamicenvironment. However, they

work fine in controlled setting. Automatic license plate recognition (LPR) while not human intervention or influence is of nice importance inside the sector of image method and pattern recognition.

Though twenty years are spent for rising LPR systems in step with things, there are still several challenges in achieving high detection and recognition rates. One such issue is quality have an effect on ted by the subsequent reasons:

i. Severe outside illumination conditions through out image acquisition, when ever will expect effects from head light and sun light.

ii. Quality license plate images which frequently embrace damaged or strained license plates and non-license plate character written on vehicles, and

iii. Perspective distortions that is quite common because of distance or view point variations.

\author{
Dr. D. Gowri Shankar Reddy ${ }^{2}$ \\ ${ }^{2}$ Assistant Professor, \\ Department of Electronics and Communication \\ Engineering, \\ SV University, Tirupathi
}

To identify the recognized characters of license plate, we have used several techniques of image processing which will be obtained to get the segmented area of interest of license plate images in existing methods. Some of that steps are Data acquisition, Color conversion procedure, Image segmentation using Otsu's Thresholding method, Noise removing and image subtraction, Image cropping, Bounding field, Optical character realization established on Template matching method. When it comes to our proposed method, character is recognized with the help of Convolutional Neural Networks (CNN) and further information is given in below sections.

\section{LITERATURE SURVEY}

Tan, X.-J., JunLiu, C [1]:This paper presents a videobased resolution for real time vehicle detection and numeration system, employing a police investigation camera mounted on a comparativelyhigh place to amass the traffic video stream. The 2 main ways applied during this system are: the adaptation background estimation and therefore the mathematicianshadow elimination. The previous permits a strong moving detection especially in complex scenes. The latter is based on color space HSV, which is able to deal with different size and intensity shadows. After these two operations, it obtains an image with moving vehicle extracted, and then operation counting is effected by a method called virtual detector.

Kamat, V., Ganesan, S [2]:addressed the general problem of the detection of vehicle license plates from road scenes, for the purpose of vehicle tracking. It describes an algorithm for detecting a license plate from a road scene acquired by a CCD camera, using image processing techniques, where the authors have used the Hough transform (HT) for line detection (the shape of the license plates is defined by lines) because it fits well into this application as it facilitates the selection of lines from specific areas and specific orientations within the image. This is followed by a brief description of the image processing system on which this algorithm has been implemented. Lastly, the results and conclusions of the application of the license plate detection algorithm to a real vehicle are discussed.

Yanamura, Y., Goto, M., Nishiyama, D [3]:In this paper, a new methof is proposed for license plate extracting and tracking from time sequential images taken by a video 
camera on a vehicle. For extracting and tracking the plate correctly, we use Hough transform and Voted Block Matching. This method enables the extracting and tracking even when the distance between the camera on the vehicle and the license plate of another vehicle is changing or the plate is occluded by some objects.

Martin, F., Garcia, M., Alba, L [4]: VLP (Vehicle License Plates) peruse using PC visionis realized.. Plate region relies upon numerical morphology and character affirmation is realized using partitioned.

Hongliangand, B., Changping, L [5]: This work hybrid license plate extraction count in light of the edge bits of knowledge and morphology for watching the interstate ticketing systems. The procedure can upgrade the region rate just by the edge estimations. The proposed approach can be disconnected into four

segments, which are, vertical edge

acknowledgment, edge quantifiable examination, dynamic based label zone, and morphology-based label extraction. The count can quickly and adequately perceive the zone of vehicle plates.

Zheng, D., Zhao, Y., Wang, J [6]: presents a steady and lively method for license plate area. License plate area contains rich edge and surface information. We first think out the vertical edges of the car image using image change and Sobel head, by then remove most of the establishment and hullabaloo edges by a fruitful estimation, finally glance through the plate area by a square shape window in the remaining edge image and bit the plate out from the principal car image. Preliminary occurs display the significant healthiness and capability of our procedure.

\section{EXISTING METHOD}

The following block diagram explains the process of vehicle plate detection using existing method.

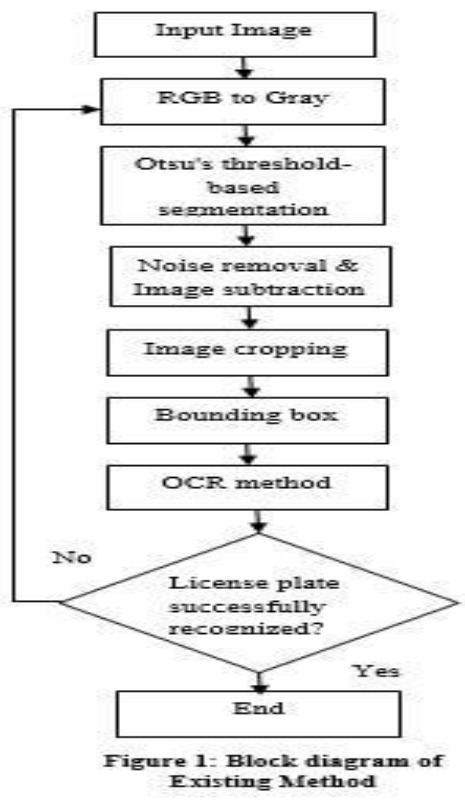

\section{- Acquisition:}

The info acquisition procedure is done through obtaining snap shots of autos. On this venture, graphics of a total of 14 automobiles were captured for evaluation approach.

\section{$>$ Color Conversion Procedure:}

RGB image is changed into grayscale picture. Furthermore, the RGB photo is also intricate to be segmented. As a consequence, the captured portraits are modified from RGB picture to grayscale photograph to supply an foremost picture for the Otsu's thresholding segmentation approach. This conversion is required to reduce the complexity of an image.

\section{Image segmentation using Otsu's thresholding:}

The transformed grayscale image will probably be processed utilizing Otsu's thresholding manner. Otsu's thresholding is a procedure used in image segmentation to automatically calculate a threshold price utilizing an iterative system. It additionally controls the superb threshold value through maximizing the overall variance between the background and object.

\section{Noise removing and image Subtraction:}

This step is foremost with a purpose to get rid of the small spots and undesired objects in the image. These small spots and undesired objects will likely be removed to increase the accuracy of realization process. In the beginning, these undesired objects are removed with the aid of making use of the neighborhood growing system. The important thing usefulness of subtraction is the enhancement of change between pictures which result a desired picture for segmenting the registration number plate.

\section{> Image Cropping for the segmented license plate:}

This approach is perform in order to crop the segmented license plate by means of utilising the standardize values. The photo cropping is completed in keeping with the desired attitude and distance between the registration code and digital camera of the enter photo.

\section{> Bounding field:}

As a result, the implication of cognizance methods begin with making a choice on the alphabet and quantity from the historical past making use of bounding field function. The bounding field is created on the snapshot the place the approach detects the presence of object.

\section{- Optical character realization established on Template matching method:}

OCR is a process to identify the information from the segmented registration code. This system used to respect printed information on an image to provide an output data along with characters. The procedure is done with the aid of analyzing the bounding field on the images and create algorithm to evaluate the similarity of theobtained personal with the educational knowledge. 


\section{- Disadvantages}

The two main drawbacks of this method are:

$>$ The preference for rounded regions, which are of less interest in typical images.

- The lack of affine invariance in the presence of blur.

Low accuracy in detection and recognition.

\section{PROPOSED METHOD}

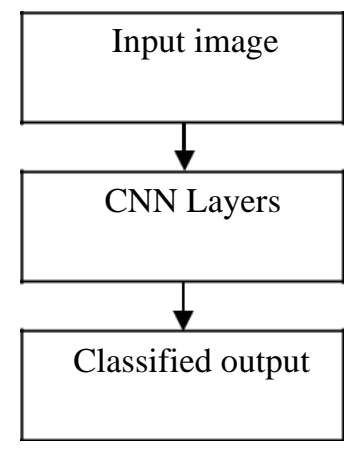

Figure 2: Simple block diagram of proposed method

\section{- Convolutional Neural Network:}

A convolutional neural network (CNN or ConvNet) is one of the most popular algorithms for deep learning, a type of machine learning in which a model learns to perform classification tasks directly from images, video, text, or sound. CNNs are particularly useful for finding patterns in images to recognize objects, faces, and scenes. They learn directly from image data, using patterns to classify images and eliminating the need for manual feature extraction. In a regular Neural Network there are three types of layers:

1. Input Layers: It's the layer in which we give input to our model. The number of neurons in this layer is equal to total number of features in our data (number of pixels in case of an image).

2. Hidden Layer: The input from Input layer is then feed into the hidden layer. There can be many hidden layers depending upon our model and data size. Each hidden layers can have different numbers of neurons which are generally greater than the number of features. The output from each layer is computed by matrix multiplication of output of the previous layer with learnable weights of that layer and then by addition of learnable biases followed by activation function which makes the network nonlinear.

3. Output Layer: The output from the hidden layer is then fed into a logistic function like sigmoid or softmax which converts the output of each class into probability score of each class.

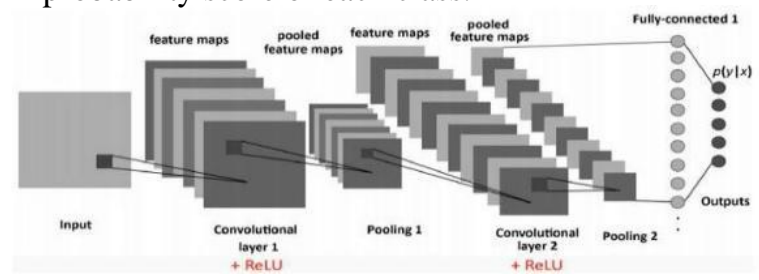

Figure 3: Block diagram of $\mathrm{CNN}$ layers

\section{Convolution Layer:}

The convolution layer is the core building block of the CNN. It carries the main portion of the network's computational load. This layer performs a dot product between two matrices, where one matrix is the set of learnable parameters otherwise known as a kernel, and the other matrix is the restricted portion of the receptive field. The kernel is spatially smaller than an image, but is more in-depth. This means that, if the image is composed of three (RGB) channels, the kernel height and width will be spatially small, but the depth extends up to all three channels.

\section{Pooling Layer:}

The pooling layer replaces the output of the network at certain locations by deriving a summary statistic of the nearby outputs. This helps in reducing the spatial size of the representation, which locate the ROI from the resulted image of the image masking phase, Sauvola Binarization technique has been conducted. It decreases the required amount of computation and weights. The pooling operation is processed on every slice of the representation individually.

\section{$>$ Fully Connected Layer:}

Neurons in this layer have full connectivity with all neurons in the preceding and succeeding layer as seen in regular FCNN. This is why it can be computed as usual by a matrix multiplication followed by a bias effect. The FC layer helps map the representation between the input and the output.

\section{Non-Linearity Layers:}

Since convolution is a linear operation and images are far from linear, non-linearity layers are often placed directly after the convolutional layer to introduce non-linearity to the activation map. There are several types of non-linear operations, the popular ones being:

\section{Sigmoid}

The sigmoid non-linearity has the mathematical form $(\quad)=$ $1 /(1+-)$. It takes a real-valued number and "squashes" it into a range between 0 and 1 .

\section{Tanh}

Tanh squashes a real-valued number to the range $[-1,1]$. Like sigmoid, the activation saturates, but-unlike the sigmoid neurons - its output is zero centered.

\section{3. $\operatorname{ReLU}$}

The Rectified Linear Unit (ReLU) has become very popular in the last few years. It computes the function $f($ ) $=(0, \quad)$. In other words, the activation is simply threshold at zero. 


\section{- Applications:}

- Object detection

- Semantic segmentation

- Image captioning

\section{> Advantages:}

- The processing time will be less

- Best results will be experienced.

\section{EXPERIMENTAL RESULTS}

Existing Method Results:

\section{input image}

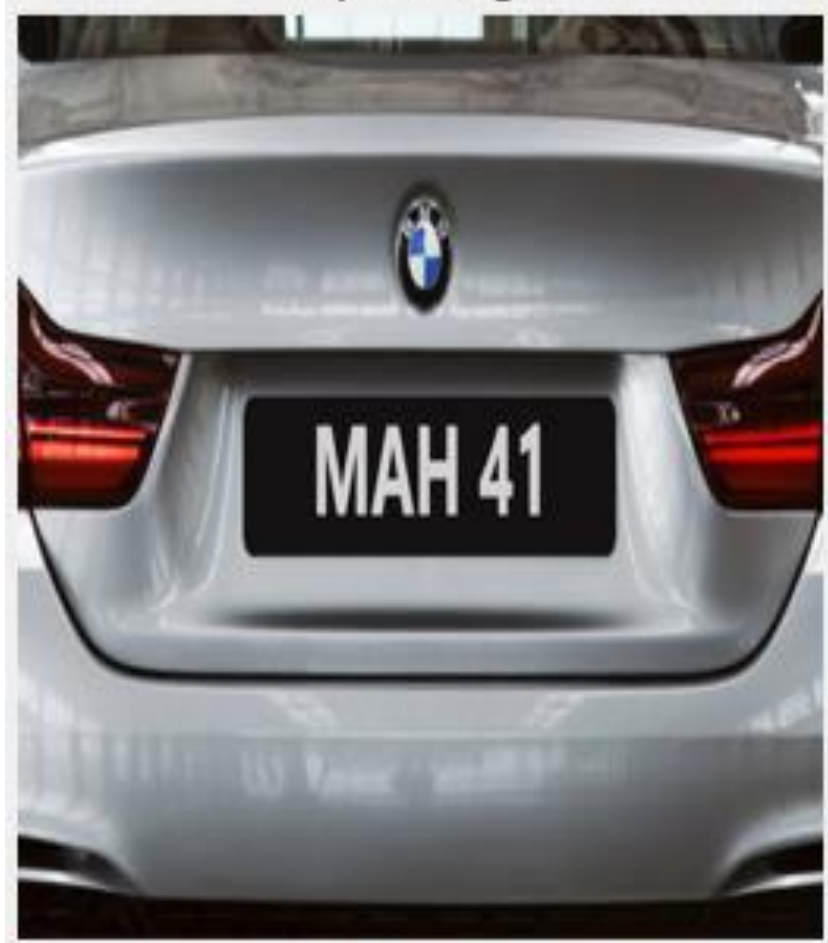

Figure 4: Input image

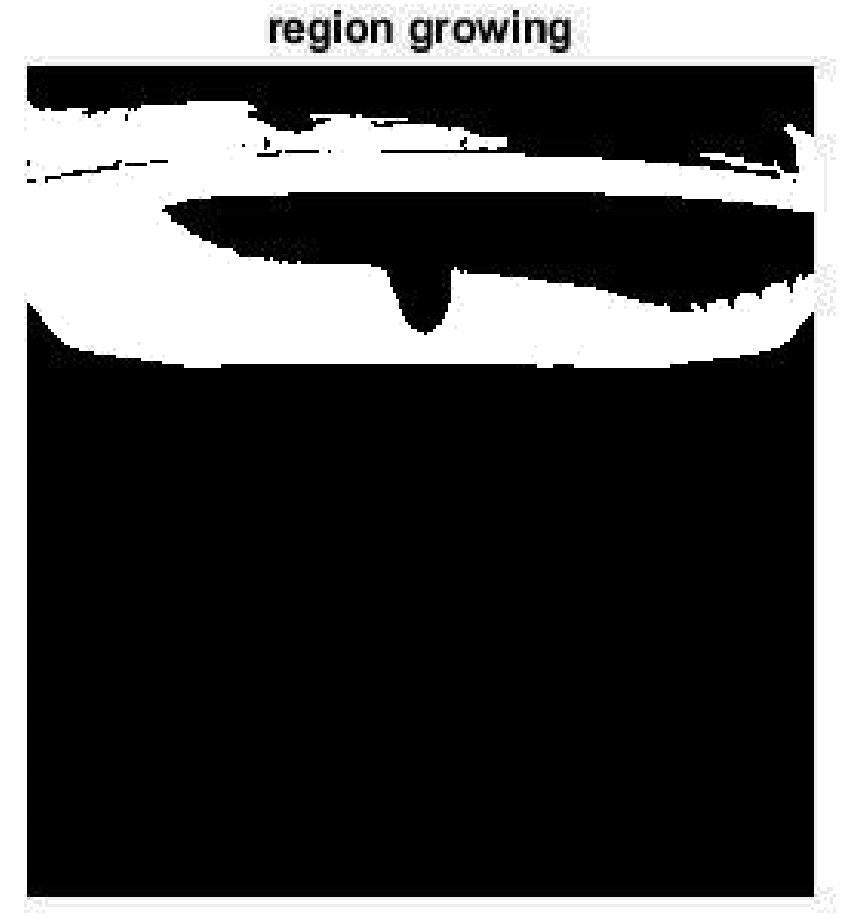

Figure 5: Region Growing

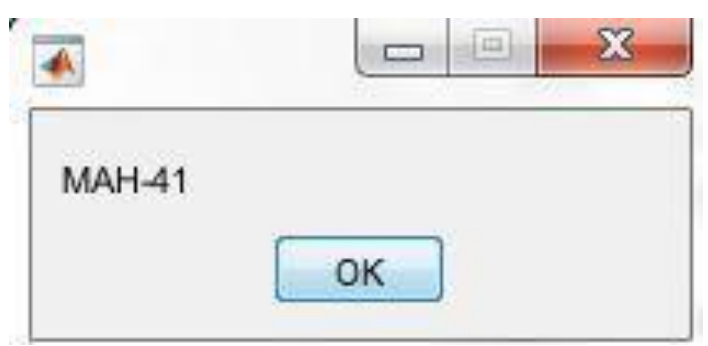

Figure 6: Classified output

Command Window

Accuracy $=$

44.7360

Figure 7: Accuracy 


\section{Proposed Method Results:}

1) VEHICAL PLATE OF CAR-A

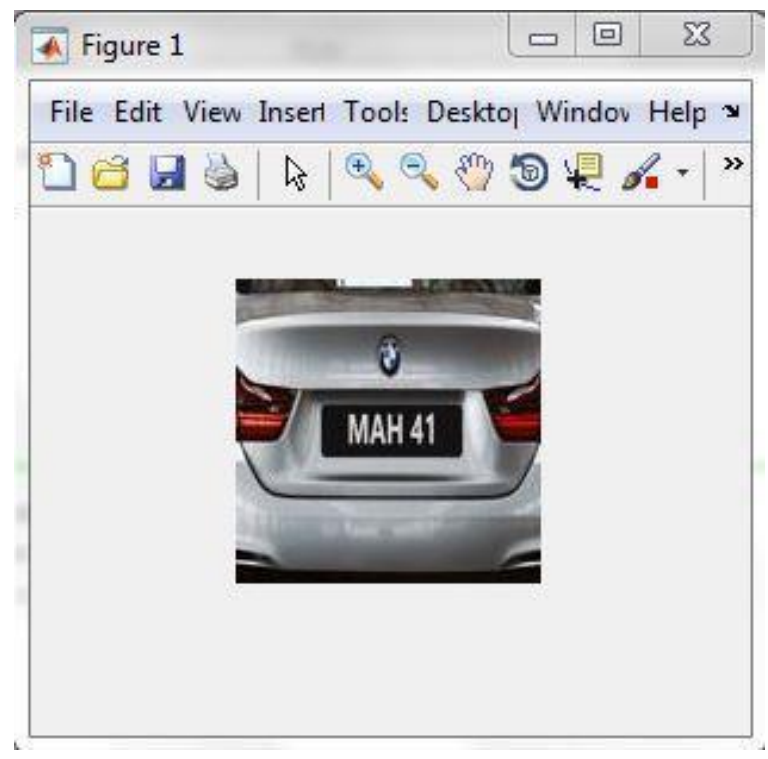

Figure 8: Input image

Training on single CPD.

Initializing inage normalization.

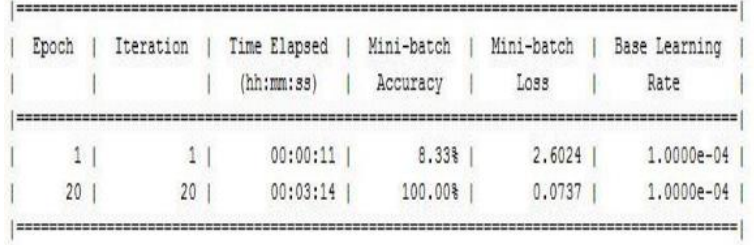

Figure 9: Training process

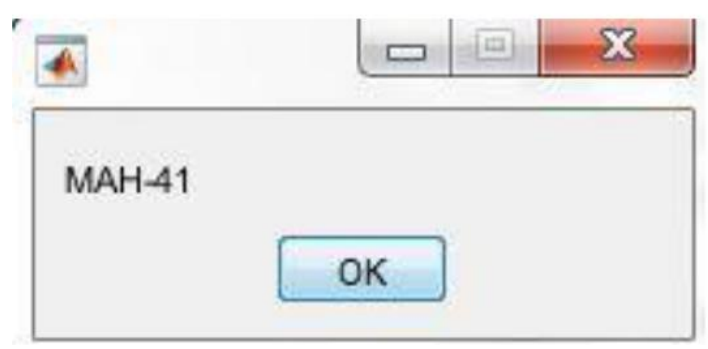

Figure 10: Classified output

\section{2) VEHICAL PLATE OF CAR-B}

Figure 1

File Edit View Insert Tools Desktol Windor Help s

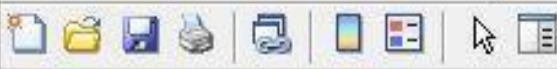

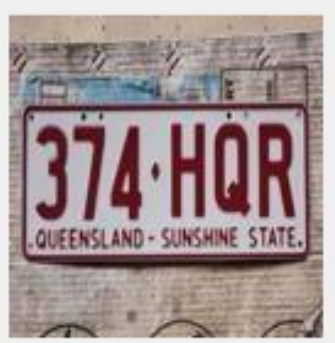

Figure 11: Input image

\begin{tabular}{|c|c|c|c|c|c|}
\hline 1 & 1 & $00: 00: 10$ & 1 & 5426 & \\
\hline 20 & 20 & $00: 02: 59$ & 100.008 & $-0,0000 e+00$ & $1.0000 \mathrm{e}-04$ \\
\hline
\end{tabular}

Figure 12: Training process

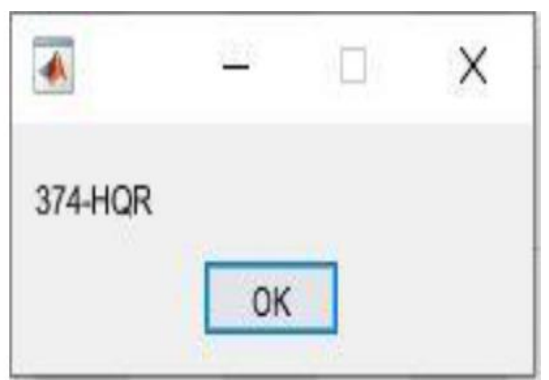

Figure 13: Classified output 


\section{CONCLUSION}

Finally, Improved the character recognition of blurred and obscure image Convolutional Neural Network (CNN). This method implements an efficient license plate recognition system that first detects vehicles and then retrieves license plates from vehicles to reduce false positives on plate detection. The experimental results show that the proposed model was capable enough to successfully detect the vehicle number plate from images that contain natural scene and different lighting conditions.

\section{REFERENCES}

[1] Tian, B., Yao, Q., Gu, Y., Wang, K., Li, Y.: Video processing techniques for traffic flowmonitoring: a survey. In: 14th International IEEE Conference on Intelligent Transportation Systems (ITSC), pp. 1103-1108 (2011)

[2] Barcellos, P., Bouvie, C., Escouto, F.L., Scharcanski, J.: A novel videobased system fordetecting and counting vehicles at user-defined virtual loops. Expert Syst. Appl. 42, 1845-1856 (2015)

[3] Tan, X.-J., JunLiu, C.: A video-based real- time vehicle detection method by classifiedbackground learning. World Trans. Eng. Technol. Edu. 6, 189 (2007)

[4] Anagnostopoulos, C., Anagnostopoulos, I., Tsekouras, G., Kouzas, G., Loumos, V.,Kayafas, E.: Using sliding concentric windows for license plate segmentation andprocessing. In: IEEE Workshop on Signal Processing Systems Design and Implementation,pp. 337-342, November 2005

[5] Anagnostopoulos, C., Anagnostopoulos, I., Loumos, V., Kayafas, E.: A licenseplate- recognition algorithm for intelligent transportation system applications. IEEE Trans.Intell. Transp. Syst. 7(3), 377-392 (2006)

[6] Sonka, M., Vaclav H., Boyle, R.D.: Mathematical morphology. Image processing, analysis, and machine vision. In: International Student edn. Thompson Learning, Toronto, pp. 657-664 (2008).

[7] S. Yu, B. Li, Q. Zhang, C. Liu and M. Q. H. Meng, A novel license plate location method based on wavelet transform and EMD analysis, Pattern Recognition, Vol. 48, Issue. 1, 2015, pp 114-125.

[8] V. Tadi, M. Popovic and P. Odry, Fuzzifiedgabor filter for license plate detection, Vol. 48, EAAI, 2016, pp 40-58.

[9] J. Tian, R. Wang, G. Wang, J. Liu and Y. Xia, A two-stage character segmentation method for Chinese license plate, Computers and Electrical Engineering, Vol. 46, 2015, pp 539553.

[10] C. N. E. Anagnostopoulos, L. E. Anagnostopoulos, V. Loumos and E. Kayafas, A license plate-recognition algorithm for intelligent transportation system applications, IEEE Trans. ITS, Vol. 7, Issue. 3, 2006, pp 377-392.

[11] K. Suresh, G. M. Kumar and A. N. Rajagoplan, Superresolution of license plates in real traffic videos, IEEE Trans ITS, Vol. 8,Issue. 2, 2007, pp 321-331.

[12] S. Du, M. Ibrahim, M. Shehata and W. Badawy, Automatic license plate recognition (ALPR): A state-of-the-art review, IEEE Trans. CSVT, Vol. 23, Issue. 2, 2013, pp 311-325.

[13] V. Khare. P. Shivakumara, P. Raveendran, L. K. Meng and H. H. Woon, A new sharpness based approach for character segmentation in license plate images, In Proc. ACPR, 2015, pp. 544-548.

[14] B. Epshtein, E. Ofek, Y. Wexler, Detecting text in natural scenes with stroke width transform. In: Proc. CVPR, 2010, pp 2963-2970.

[15] N. R. Howe, Document binarization with automatic parameter tuning, IJDAR, Vol. 16, Issue. 3, 2013, pp 247-258.

[16] S. Roy, P. Shivakumara, P. P. Roy, U. Pal, C. L. Tan and Tong $\mathrm{Lu}$, Bayesian classifier for multi-oriented video text recognition system. Expert System with Applications, Vol. 42, Issue. 13, 2015, pp 5554-5566.
[17]Latha, M.G., Chakravarthy, G.: An improved Bernsen algorithm approaches for license platerecognition. IOSR-JECE: IOSR J. Electron. Commun. Eng. 3(4), 01-05 (2012)

[18] Wang, T.-H., Ni, F.-C., Li, K.-T., Chen, Y.-P.: Robust license plate recognition based ondynamic projection warping. In: Proceeding

[19] IEEE International Conference Networking,Sensing and Control, pp. 784-788 (2004). 